\title{
Measurement of the antiproton-nucleus annihilation cross-section at very low energies
}

\author{
M. Corradini • M. Hori • M. Leali • E. Lodi Rizzini • \\ V. Mascagna $\cdot$ A. Mozzanica $\cdot$ M. Prest $\cdot$ E. Vallazza $\cdot$ \\ L. Venturelli • N. Zurlo
}

Published online: 22 September 2009

(C) Springer Science + Business Media B.V. 2009

\begin{abstract}
The main fields of investigation of the ASACUSA Experiment at the Antiproton Decelerator at CERN concern test of CPT violation through spectroscopy of antihydrogen and antiprotonic helium, atomic collision and nuclear physics experiments. The nuclear physics program foresees measurements of $\bar{p}$ nuclei cross sections at low energies (from few $\mathrm{MeV}$ down to the $\mathrm{keV}$ region) using a cylindrical detector with 6 layers of scintillating fibers surrounding the target. These measurements will contribute to understand the dynamic of the annihilation process and in particular to explain why the antiproton-nucleus annihilation rate at very low energy appears independent from the size of the target, as measured by the Obelix Collaboration at CERN for H, D and He. The preliminary results of the measurements performed during 2008 are presented.
\end{abstract}

Keywords Antiproton • Annihilation • Cross section • Nuclear

M. Corradini $(\bowtie) \cdot$ M. Leali $\cdot$ E. Lodi Rizzini · V. Mascagna · A. Mozzanica ·

L. Venturelli $\cdot$ N. Zurlo

Dipartimento di Chimica e Fisica per l'Ingegneria e per i Materiali,

Università di Brescia, 25133 Brescia, Italy

e-mail: corradini@bs.infn.it

M. Corradini · M. Leali · E. Lodi Rizzini · V. Mascagna · A. Mozzanica · L. Venturelli · N. Zurlo Istituto Nazionale di Fisica Nucleare, Gruppo Collegato di Brescia, 25133 Brescia, Italy

M. Hori

Max-Planck-Institut fur Quantenoptik, Hans-Kopfermann-Strasse 1, 85748 Garching, Germany

M. Hori

Department of Physics, University of Tokyo, 7-3-1 Hongo, Bunkyo-ku, Tokyo 113-0033, Japan

M. Prest · E. Vallazza

Dipartimento di Scienze Fisiche e Matematiche, Università di Como, 22100 Como, Italy

M. Prest · E. Vallazza

Istituto Nazionale di Fisica Nucleare, Sezione di Trieste, 34127 Trieste, Italy 


\section{Introduction}

The antiproton $(\bar{p})$ physics continues to play an important role in improving the knowledge of both the fundamental interactions and the structure of the matter. Since its discovery [1], that made possible to extend the concept of matter-antimatter symmetry from the elementary particles like electron and positron also to composite particles (since proton and antiproton consist of 3 quarks and antiquarks), several fundamental results have been achieved. Recently the discovery of the top quark, the formation of the first cold antihydrogens and the start of the search of the Higgs boson at LHC have been possible thanks to the availability of antiproton beams of both very high and very slow energy.

In low-energy antiproton physics the annihilation process is one of the most fascinating since here the matter experiences a transition from its baryon phase to one consisting solely of mesons. The measurement of the annihilation cross section of slow antinucleons on nuclei can help in the determination of the potential parameters, in the search of nuclear resonances, in the investigation of the excitation process of the nuclear matter following the annihilation and in the astrophysics search for antimatter in the universe.

A considerable amount of data at low energy has been acquired in the 80's and 90's at LEAR at CERN and a discrepancy from the behavior at high energies has been detected. In light nuclei $\left(\mathrm{H}, \mathrm{D},{ }^{4} \mathrm{He}\right)$ the antiproton annihilation cross sections $\left(\sigma_{\text {ann }}\right)$ at $\bar{p}$ momentum below $60 \mathrm{MeV} / \mathrm{c}$ have comparable values, whereas at higher momenta $(>100 \mathrm{MeV} / \mathrm{c})$ the $\bar{p}$-nucleus $\sigma_{a n n}$ increases regularly with the number of nucleons in the nuclei [2-4]. Also low statistics data on ${ }^{20} \mathrm{Ne}$ at $57 \mathrm{MeV} / \mathrm{c}$ [5] and on ${ }^{3} \mathrm{He}$ [6] seem to confirm a saturation effect of $\sigma_{\text {ann }}$ with the mass number $A$ at low energy. The situation is different when as a projectile it is used an antineutron: the $\mathrm{A}^{2 / 3}$ scaling law is valid even down to $50 \mathrm{MeV} / \mathrm{c}$ [7]. These results together with the data of widths and shifts of spectroscopic transitions in antiprotonic atoms $[8,9]$ have stimulated different model analysis where huge saturation effects dominate the $\bar{p}$-nucleus interaction $[10,11]$.

In order to get new information, the ASACUSA Collaboration has started a systematic study of antiproton annihilation cross sections with heavier nuclei and at lower energy [12].

The preliminary results of the measurements performed with $5 \mathrm{MeV}$ antiprotons on solid target (Mylar, Ni, Sn, Pt) are presented.

\section{The experiment}

The ASACUSA Experiment is carrying out at CERN's Antiproton Decelerator facility (AD) a wide investigation program which is mainly focused on tests of CPT violation, through the spectroscopy of antihydrogen and of antiprotonic helium, but which also includes the study of atomic collisions and of nuclear physics [12].

The nuclear physics program concerns the measurements of $\bar{p}$ annihilation cross sections on different nuclei and at different energies from the $\mathrm{MeV}$ down to the $\mathrm{keV}$ region.

The Antiproton Decelerator [13] is the successor of LEAR and is presently the only existing source of low energy antiprotons. The beam from AD is $100 \mathrm{~ns}$ long, 


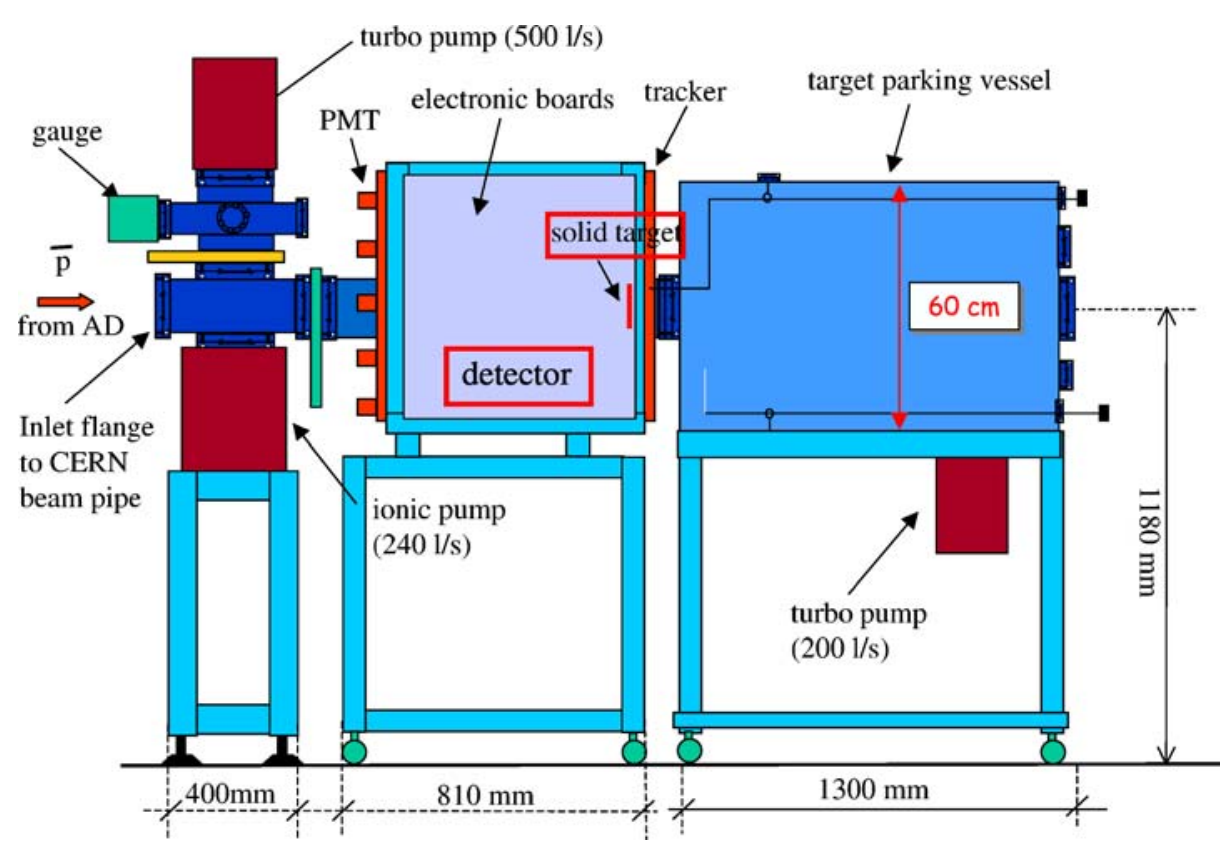

Fig. 1 ASACUSA experimental set-up for $\sigma_{a n n}$ measurement of $\bar{p}$ at $5 \mathrm{MeV}$

contains $2-4 \times 10^{7} \bar{p}$ s of momentum $p=100 \mathrm{MeV} / \mathrm{c}$ (corresponding to energy 5.3 $\mathrm{MeV}$ ) with a $\Delta p / p=0.01 \%$ and an emittance $0.3 \pi \mathrm{mm}$ mrad.

The main difficulty in performing a measurement of antiproton $\sigma_{a n n}$ at $\mathrm{AD}$ comes from the pulsed characteristic of the antiproton beam, since even a small number of annihilations can saturate the acquisition system.

To overcome this problem the solution chosen by ASACUSA foresees both accurate settings of the AD beam and a particular design of the experimental set-up.

As for the AD beam optimization, the so called "multiple extraction" option was used: the $100 \mathrm{MeV} / \mathrm{c}$ beam was divided into 6 bunches with lower intensity which were kicked out individually separated by $2.4 \mathrm{~s}$.

In addition, for each bunch the time gate of the $\mathrm{AD}$ septum was reduced to remove the long tails (various hundreds of nanoseconds) of very low intensity (some thousands of antiprotons) that blinded our detector before the arrival of the signal. The final actual length of each bunch resulted to be $40-50 \mathrm{~ns}$, as required. Moreover the radial halo of the beam was reduced to few $\mathrm{mm}$, by moving the AD scrapers position, so avoiding any annihilation on the lateral wall of the target vessel when the target was removed (see after).

The experimental set-up was designed with a particular care to avoid contaminations from the $\bar{p}$ annihilations not occurring on the target. The experimental set-up is sketched in Fig. 1. It is quasi-axial symmetrical around the $\bar{p}$ beam and consists of a vessel containing a thin solid target surrounded by a vertex detector.

The vessel was directly connected to the AD beam line without any material along the $\bar{p}$ path before the target. To reduce the Rutherford scattering background the radius of the target vessel was increased after the target. The target vessel was long 
enough to reduce contaminations from the beam annihilations on the end wall, where a beam counter was placed to monitor the $\bar{p}$ beam intensity.

The selection of the annihilation events on the target was performed by means of a vertex detector that was able to reconstruct the tracks of the charged pions emitted in the $\bar{p}$ annihilation. Since the tracks coming from different annihilations can be disentangled only if few annihilations occur per bunch, the targets must be very thin.

The vertex detector is made with scintillating fibers in order to fulfill the requirements of both good time and spatial resolution [14, 15].

It consists of two cylindrical shells arranged coaxially to the $\bar{p}$ beam axis. They are $50 \mathrm{~cm}$ long with external radius of 11.5 and $15 \mathrm{~cm}$, respectively. Each shell contains 3 double-layers of scintillating fibers: one is parallel to the $\bar{p}$ beam, the other two are placed to form helices with \pm 20 degrees. This permits to measure 2 points for each track crossing the detector and to resolve the ambiguities when several tracks coexist.

Every double-layer is made by scintillating fibers of $1 \mathrm{~mm}$ diameter which are collected in bunches of 4 neighboring fibers. Each bunch is connected to a channel of a photomultiplier (PMT). This solution is more convenient in respect to use single fiber of $2 \mathrm{~mm}$ diameter since the scintillating material thickness is more uniform with a reduction of the empty space too. The fibers are Bicron BCF10 Multiclad with EMA (Extra Mural Absorber, i.e. a coating that eliminates the crosstalk between adjacent fibers) and are read out by 64-channels multianode PMTs H75468 by HAMAMATSU.

The signals from the PMTs are amplified, shaped and discriminated by VLSI ASICs manufactured by IDEAS (VA64TAP2.1 and LS64) and then are sent to a Cyclone II FPGA by Altera for time sampling. The sampled data are sent to the VME-based DAQ. The total number of channels is 2688 for a total length of fibers of almost $10 \mathrm{~km}$. The time resolution of the DAQ chain was measured on a prototype and resulted to be of the order of some nanoseconds with an efficiency of $94 \%$.

The design of the apparatus has been driven by Monte Carlo simulations based on Geant 3.21 and a combinatorial algorithm for the vertex determination.

In the simulation program a phase space generator for antiproton-proton annihilation is inserted with the branching ratios taken from [16].

The vertex is determined by considering all the possible straight lines passing through 2 hits (one of the inner shell of the detector, the other of the external one). Some of these lines correspond to charged pion tracks, while the others are fake tracks coming out from the combinatorial logic of the used algorithm. For each couple of tracks the point of the minimum distance between them is measured. The average position of these points is then evaluated to determine the final annihilation vertex.

The efficiency in the vertex determination is $80 \%$ for the simulated antiprotonproton annihilations occurring at the centre of the apparatus, assuming no inefficiency in the fiber detection system. The spatial resolutions is $0.4-0.5 \mathrm{~cm}$ on the $\mathrm{z}$-coordinate (z-axis is along the $\bar{p}$ beam direction) and $0.3-0.4 \mathrm{~cm}$ on the $\mathrm{x}, \mathrm{y}$ transverse coordinates.

The targets are metallic disks ( $5 \mathrm{~cm}$ in diameter) sputtered on Mylar foils $(12 \mathrm{~cm}$ in diameter and 0.9 micrometers in thickness). We used nickel, tin, platinum targets with suitable thickness (hundreds of nanometers) in order to have annihilation events numbers comparable with the ones from Mylar (assuming an $\mathrm{A}^{2 / 3}$ law for $\sigma_{\text {ann }}$ ). The Mylar in turn constitutes a target. 
Fig. 2 Time distributions of the hits measured in one layer of the detector for one spill with different targets
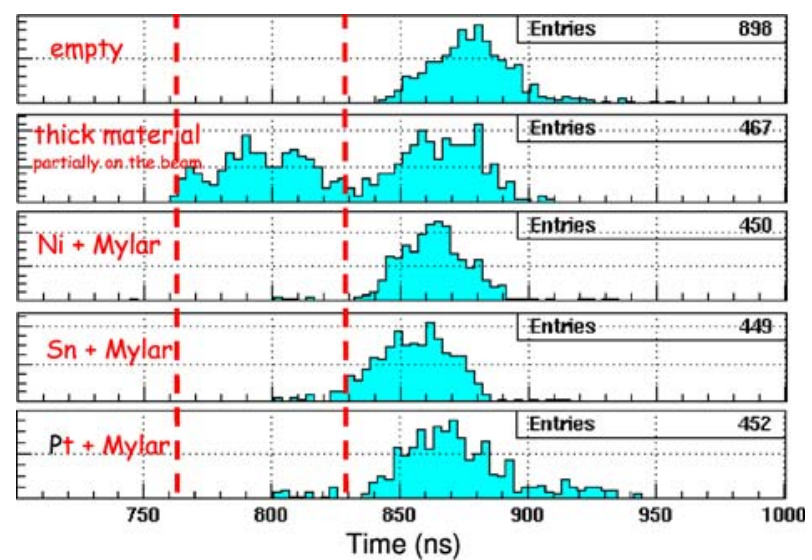

One target at a time was placed along the $\bar{p}$ beam near the end of the smaller part (inner radius $\mathrm{R}=7.5 \mathrm{~cm}$ ) of the target vessel. The swap of target was obtained very quickly without opening the target vessel by means of a rotarylinear multi-motion system which moved the selected target on the $\bar{p}$ beam from the parking inside the wider part (inner radius $\mathrm{R}=30 \mathrm{~cm}$ ) of the target vessel (see Fig. 1).

We present here the preliminary analysis of a part of the data acquired in one week ( 8 h per day) in July 2008 by means of the described apparatus. In 2006 and 2007 comparable acquisition times were used to understand and to improve the AD settings and to modify the experimental set-up to increase the background rejection.

In Fig. 2 we show typical time distributions of the measured hits in one layer of the detector with different targets for one antiproton bunch delivered by AD. The signals from the $\bar{p}$ annihilations in the targets are expected in the interval $\Delta t$ between 760 and 830 ns.

This clearly appears if we compare in Fig. 2 the presence of few hits with $\mathrm{Ni}, \mathrm{Sn}$ and Pt targets with the absence of any hit when no target is placed on the $\bar{p}$ beam (the so called "empty target"). It is confirmed further by the lot of hits in $\Delta t$ when a dummy tick target is inserted.

After the aforementioned interval $\Delta t$ a flux of pions coming from the end wall of the target vessel fires the whole detector.

The absence of events before $830 \mathrm{~ns}$ with the empty target also indicates that the radial halo of $\bar{p}$ beam is well confined and that the annihilations from the end wall of the target vessel are well-separated in time from the annihilations in the target.

For each target the number of the reconstructed vertices has been counted and the contribution of the Mylar support has been subtracted by using the data acquired with a reference target made only by a Mylar foil. The antiproton annihilation cross sections can be determined by means of the following formula:

$$
\sigma_{a n n}=\frac{N_{e v}}{N_{c} \cdot N_{\bar{p}} \cdot \rho \cdot \frac{N_{A}}{M} \cdot l \cdot \epsilon_{e v}}
$$

where $N_{e v}$ is the number of vertices reconstructed in the target, $N_{\bar{p}}$ is the incident $\bar{p}$ s on the target, $N_{c}$ is the number of scattering centers of the target molecule $\left(N_{c}=1\right.$, apart for Mylar), $\rho$ is the target density, $N_{A}$ is the Avogadro's number, $M$ is the 
Fig. 3 Antiproton $\sigma_{a n n}$ data with the best fit function: in a with only the reconstructed vertices, in b also with the events with single track crossing the target (see text for the explanation)
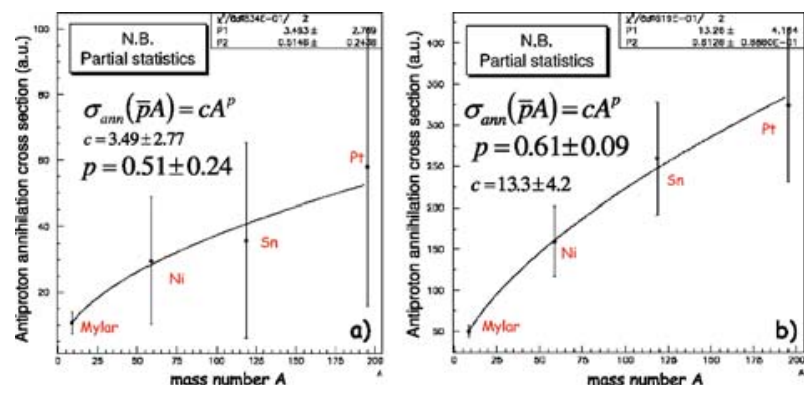

molecular weight of the target, $l$ is the target thickness, $\epsilon_{e v}$ is the vertex detection efficiency.

In the Mylar case the number of annihilations is determined by the weights of the $\mathrm{H}, \mathrm{C}$ and $\mathrm{O}$ elements in the annihilation dynamics: the estimation of the average $\sigma_{a n n}$ value has been related to the average mass number of Mylar. Due to the relatively large statistical error of the measured events number this assumption does not affect strongly the estimation of the annihilation cross section with A.

The function $\sigma_{0} \mathrm{~A}^{\alpha}$ has been fitted to the $\sigma_{a n n}$ data. In Fig. 3a the data and the best fit function are plotted. The resulting free parameter $\alpha=0.51 \pm 0.24$ indicates that within uncertainties the $\mathrm{A}^{2 / 3}$ dependence of the $\bar{p}$ annihilation cross section is compatible with the data.

The statistical errors shown in Fig. 3a will be reduced in the final analysis by using all the available data. A further reduction can be done by adding the events with only one track. For the determination of a vertex at least 2 reconstructed tracks are needed. This represent a limit in the counting of the annihilation events since in the present measurement the target is axially near the edge of the detector where the efficiency in the vertex reconstruction is reduced. In Fig. $3 b$ we present the data where the events with single track crossing the target are added to the reconstructed vertex events used in Fig. 3a. The behavior of the $\sigma_{a n n}$ values is still in agreement with the $\mathrm{A}^{2 / 3}$ law since the $\alpha$ free parameter results to be $0.61 \pm 0.09$.

\section{Conclusions}

For the first time the relative $\bar{p}$ annihilation cross sections on not-light nuclei (Mylar, $\mathrm{Ni}, \mathrm{Sn}, \mathrm{Pt})$ have been measured at low energy $(5 \mathrm{MeV})$ by the ASACUSA Collaboration. This represents also the first measurements of antinucleon annihilation cross section at low energy performed at a pulsed beam.

Preliminary results indicate that $\sigma_{a n n}(\bar{p}-A) \propto \mathrm{A}^{2 / 3}$ is compatible with the data. This suggests that the saturation effect seen with light nuclei at lower energies (below $60 \mathrm{MeV} / \mathrm{c}$ ) is not present for the measured target at $100 \mathrm{MeV} / \mathrm{c}$. Data analysis is still in progress to confirm this preliminary result. Starting from 2009 an extension of the measurement down to $<100 \mathrm{keV}$ is foreseen with the addiction of the ASACUSA radio frequency decelerator (RFQD) and with very thin targets. 


\section{References}

1. Chamberlain, O., et al.: Phys. Rev. 100, 947 (1956)

2. Bertin, A., et al.: Phys. Lett. B 369, 77 (1996)

3. Zenoni, A., et al.: Phys. Lett. B 461, 405 (1999)

4. Zenoni, A., et al.: Phys. Lett. B 461, 413 (1999)

5. Bianconi, A., et al.: Phys. Lett. B 481, 194 (2000)

6. Bianconi, A., et al.: Phys. Lett. B 492, 254 (2000)

7. Bressani, T., et al.: Phys. Rep. 383, 213 (2003)

8. Augsburger, M., et al.: Nucl. Phys. A 658, 149 (1999)

9. Gotta, D., et al.: Nucl. Phys. A 660, 283 (1999)

10. Gal, A., et al.: Phys. Lett. B 491, 219 (2000)

11. Batty, C.J., et al.: Nucl. Phys. A 689, 721 (2001)

12. ASACUSA Collaboration, CERN/SPSC 2005-002, SPSC P-307 Add.1

13. Maury, S.: Hyperf. Interact. 43, 109 (1997)

14. Mozzanica, A., et al.: Nuovo Cimento B 122, 759 (2007)

15. Bolognini, D., et al.: Nucl. Instr. Meth. A 572, 281 (2007)

16. Armenteros, R., French, B.: In: Burhop, E.H. (ed.), $\bar{N} N$ Interactions in High Energy Physics, vol. 4, p. 237. Academic, New York (1969) 